\title{
Research Paper \\ Concept Analysis of Thirst as a Symptom and Its Causes From the Perspective of Iranian Traditional Medicine: A Hybrid Model Study
}

\author{
Parisa Jafari ${ }^{1} \odot$, Morteza Mojahedi ${ }^{2} \odot$, Fatemeh Hakimi ${ }^{1} \odot$, Mojgan Tansaz ${ }^{1}$, Mina Movahhed ${ }^{1} \odot$, Rasool Choopani ${ }^{1} \odot$, \\ Mahmood Khodadoost ${ }^{1} \odot$, Reza llkhani ${ }^{1} \odot$, Elham Parsa ${ }^{1} \odot{ }^{*}{ }^{*}$ Roshanak Mokaberinejad $^{1}$
}

\begin{abstract}
1. Department of Traditional Medicine, School of Traditional Medicine, Shahid Beheshti University of Medical Sciences, Tehran, Iran.
2. Department of Traditional Medicine, Iranian Medicine and History of Medical Sciences Research Center, Health Research Institute, Babol University of Medical Sciences, Babol, Iran.
\end{abstract}

\begin{tabular}{|c|c|}
\hline $\begin{array}{l}\text { Use your device to scan } \\
\text { and read the article online }\end{array}$ & On: Jafari P Moiahedi M Hakimi F Tansaz M Movahhed M Choonani R Khodadoost M Ilkhani R Parsa F Moka- \\
\hline 口乎回 & berinejad R. [Concept Analysis of Thirst as a Symptom and Its Causes From the Perspective of Iranian Traditional Medicine: A \\
\hline 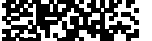 & Hybrid Model Study (Persian)]. Complementary Medicine Journal. 2020; 10(1):80-93. https://doi.org/10.32598/cmja.10.1.961.1 \\
\hline 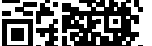 & doi'https://doi.org/10.32598/cmja.10.1.961.1 \\
\hline
\end{tabular}

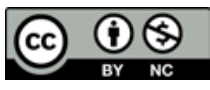

Article Info:

Received: 01 Dec 2019

Accepted: 01 Mar 2020

Available Online: 01 Jun 2020

Keywords:

Thirst, Attash, Iranian traditional medicine, Concept analysis

\section{ABSTRACT}

Objective Thirst (Attash) is a diagnostic sign of diseases from the perspective of Iranian Traditional Medicine (ITM).The purpose of this study is to provide a functional definition for the concept of thirst and its causes in ITM.

Methods In this study, we used the concept analysis (hybrid model) which has three phases; in the theoretical phase, the content related to the definition of thirst and its causes were searched in ITM books and an efficient definition of thirst was presented. In the fieldwork phase, main themes were obtained after semi-structured interviewing of 16 ITM specialists. In the final (analytical) phase, the results of the two previous phases were compared to each other to present the final definition for thirst.

Results The thirst is defined as the need for water and any food that is cold and wet, and the increase and decrease in thirst is a sign of disease. For its examination, it is necessary to pay attention to the change in the degree of thirst and the amount of consumed water or cold/wet foods. It is important to differentiate between drinking water as recommended, drinking water as habit, or drinking water because of dry mouth with thirst. Different characteristics of thirst can be used to diagnose the affected organs.

Conclusion In this study, using the concept analysis method, a functional definition of the thirst as a symptom was presented and its causes were investigated. The low number of studies on thirst and lack of access to some ITM specialists were some of the limitations of our study. It is recommended to investigate the prevalence of thirst in patients and its relationship with dystemperament in future studies.

\section{Extended Abstract}

\section{Introduction}

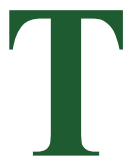

hirst (Attash in Persian) stimulates the drinking behavior [1], regulates body fluids and is an annoying symptom $[2,3]$ in patients with chronic heart failure [4], admitted to the intensive care unit [5], chron- ic obstructive pulmonary disease [6], dialysis [7], diabetes [8] and schizophrenia [9]. In Iranian tradutional medicine (ITM), the symptoms of the disease are described based on observation $[10,11]$. In conventional medicine, The Rome criteria are one of the most successful methods of using symptoms to diagnose diseases [12].

The low agreement between ITM specialists in the diagnosis of temperament (Mizaj) and dystemperament (Sue-

\section{* Corresponding Author:}

Roshanak Mokaberinejad, PhD.

Address: Department of Traditional Medicine, School of Traditional Medicine, Shahid Beheshti University of Medical Sciences, Tehran, Iran. Tel: +98 (21) 88773521

E-mail: rmokaberi@gmail.com 
mizaj) [13] shows the need to provide the same practical definitions for concepts such as signs and symptoms. Due to the importance of the symptoms of thirst in ITM and diagnosis of diseases expecially dystemperament, this study used the method of concept analysis to provide a clear and usable definition [14]for the concept of thirst [15].

\section{Materials and Methods}

A qualitative study of concept analysis was designed using a hybrid method consisting of three steps [16]. At the first phase (theoritical phase), after searching in the Noor library, keywords related to thirst and books with the most relevant content were selected based on the importance and credibility of the book and its author as well as the relevence of its texts. After dividing the texts into semantic units, the overt and covert concepts were identified. The search continued until information saturation was reached. The purpose of second step (fieldwork phase) was to investigate the new characteristics of thirst or Attash based on the clinical experiences of specialists and clarifying the characteristics mentioned in the texts by the interview method.

In this regard, interview questions were designed based on the results of previous step. ITM specialists were selected by usinga a purposive sampling method [17] with maximum diversity. In-depth, semi-structured, and face-to-face interviews continued until information saturation was achieved [18]. To achieve the main themes, after recording and typ- ing the interviews and dividing the interviews into semantic units, coding was done with MAXQAD10 software [19]. At the final step (analytical phase), by comparing the findings of first step and working on determining the differences and similarities, a practical definition of thirst was presented from the combination of the findings of the two previous steps.

\section{Results}

In ITM, thirst is defined as the need for water and any food that is cold and wet $[20,21]$; its moderate level is considered normal but its decrease and increase is a sign of illness [22]. False thirst is exacerbated by cold water and relieved by hot water, and tolerating thirst and sleeping reduces false thirst [22-29]. The time to quench your thirst after drinking water and the temperature and amonut of the water are used to determine the cause of thirst. Increased thirst is a symptom of hot mizaj and dominance of yellow bile (safra) and its decrease is a symptom of cold mizaj and dominance of phlegm [30, 31]. On the other hand, the causes of true thirst are: the need of the organs and the body for moisture, the need to eliminate dryness and abnormal heat, and the dilution of the eaten food [26].

Abnormal thirst is caused by the accumulation of concentrated salty sputum in the stomach, nature's desire to eliminate and wash it [32], factors that prevent the absorption of water into the liver, reduction of main moisture [30] and the warmth of the mouth [32]. Thirst for each body organ

Table 1. The books usied in the first step (theoritcal phase)

\begin{tabular}{|c|c|c|c|}
\hline Title & Author & Year (Hijri Shamsi) & Language \\
\hline Firdous Al-Hikmah & Ali ibn Sahl Raban Al-Tabari & $3^{\text {rd }}$ century & Arabian \\
\hline Al Tasreef Liman ‘Ajaz ‘Aan Al-Taleef & Abu Al Qasim Al Zahrawi & $4^{\text {th }}$ century & Arabian \\
\hline Kamel Al-Senaat Al-Tebieh & Alli Ibn Abbas Al-Majusi & $4^{\text {th }}$ century & Arabian \\
\hline Hidayat Al-Muta`allemin Fi al-Tibb & Al-Akhawyni Bokhari & $4^{\text {th }}$ century & Persian \\
\hline The Canon of Medicine & Ibn Sina & $5^{\text {th }}$ century & Arabian \\
\hline Commentary on Avicenna's Canon & Ibn Al-Nafis & $7^{\text {th }}$ century & Arabian \\
\hline Sharhe Asbaab-o-Alaamaat & Nafis Ibn Avaz Kermani & $7^{\text {th }}$ century & Arabian \\
\hline Bahr Al-Jawahir & Muhammad Ibn Yusuf Harawi & $10^{\text {th }}$ century & Arabian \\
\hline Kholase Al-Hekmat & Aghili Alavi Khorasani & $12^{\text {th }}$ century & Persian \\
\hline Makhzan Al-Advia & Aghili Alavi Khorasani & $12^{\text {th }}$ century & Persian \\
\hline Mizan Al-Teb & Mohammad Akbar Arzani & $10^{\text {th }}$ century & Persian \\
\hline Mofareh Al-Gholub & Mohammad Akbar Arzani & $12^{\text {th }}$ century & Persian \\
\hline Teb Al-akbar & Mohammad Akbar Arzani & $12^{\text {th }}$ century & Persian \\
\hline Eksir-E Azam & Azam Khan Chashti & $13^{\text {th }}$ century & Persian \\
\hline
\end{tabular}


Table 2. Diseases in which increased thirst was mentioned as a symptom

\begin{tabular}{|c|c|}
\hline Affected Organ & Disease \\
\hline Nose & Sarsam (meningitis), mania, qotrob (a melancholic diseases) [30]. \\
\hline Heart & Hot dystemperament of the heart [30] \\
\hline Liver & $\begin{array}{c}\text { Enlargment of the liver [30,40] when becomes hot or very cold }(30,36) \text {, Hot or cold dystemperament of } \\
\text { liver [24], obstruction within the liver and the stomach such as edema or colic [30], obstruction beneath the } \\
\text { gallbladder [47] }\end{array}$ \\
\hline Stomach & $\begin{array}{l}\text { Stomach fever, especially in the mouth [30, 32], dry dystemperament of stomach }[30,32,46] \text {, distended } \\
\text { stomach [30], Heyze (Diarrhea and vomiting) [30], the presence of salty or sweet phlegm or bile in the stom- } \\
\qquad \text { ach }[24,30,32,46] \text {, severe zarb (sprue) }[30,36]\end{array}$ \\
\hline Esophagus & hot esophagus and throat, and swelling of esophagus [30] \\
\hline Kidneys and bladder & Ziabitis, Kidney fever [30], swelling of the bladder $[30,47]$ \\
\hline Intestine & $\begin{array}{l}\text { Colic when it is caused by swelling or due to piquant (horayf) and pungent (Leza) sputums [30], hot dystem- } \\
\text { perament of jejunum [30], salty mucus accumulation in the small intestine or mesenteric arteries [46] }\end{array}$ \\
\hline Lungs & Hot dystemperament of lungs [30,46], Purulent lung injury caused by hot mizaj [35] \\
\hline Uterus & Uterine suffocation, menstrual cessation and uterine tumors [30] \\
\hline Spleen & $\begin{array}{l}\text { hot dystemperament of spleen [30], damavi (sanguine) swelling of the spleen [30, 32], safravi (choloric) swell- } \\
\text { ing of the spleen [30] }\end{array}$ \\
\hline Drugs and toxins & Eating euphorbia $[24,32]$ \\
\hline Environmental factors & $\begin{array}{l}\text { Eating snow [24, 32], actual or potential hot foods such as garlic, old wine, salty foods, sticky and slow-digest- } \\
\text { ing foods such as fish, seawater or salt water (causing heat in the mouth) }[24,30,32,36] \text {, excessive talking, } \\
\text { exercise, strenuous physical activity, sleeping after eating hot meals [30], vomiting after or during taking laxa- } \\
\text { tive }[24,30,32] \text {, viper snake bite }[30,46] \text { or eating its meat [32] }\end{array}$ \\
\hline Others & $\begin{array}{l}\text { Fullness due to yellow bile dominance [30], fever caused by yellow bile dominance (Ghab fever), sarsam } \\
\text { (meningitis) and barsam (pleurisy), jaundice, jamrah and namla [46], saher (inability to sleep) [30], excite- } \\
\text { ment caused by yellow bile dominance [47], and final stages of tuberculosis and stroke with the invovlvment } \\
\text { of the whole body such as fever [30] }\end{array}$ \\
\hline
\end{tabular}

Table 3. Diseases in which decreased thirst was mentioned as a symptom

\begin{tabular}{|c|c|}
\hline Affected Organ & Disease \\
\hline Nose & - \\
\hline Heart & - \\
\hline Liver & Cold and wet dystemperament of liver, phlegmatic and cold swelling of of liver [30] \\
\hline Stomach & $\begin{array}{l}\text { Wet stomach [47], Cold and wet dystemperament of stomach [30], shahvat-e kalbi (polyphagia), hiccups, and } \\
\text { zarb (sprue) caused by a cold stomach [32], sodavi (melancholic) swelling of stomach [22] }\end{array}$ \\
\hline Esophagus & - \\
\hline Kidneys and bladder & Cold dystemperament of kidney [22] \\
\hline Intestine & Cold dystemperament of intesine [30], phlegmatic zahir (stomach cramp) [22] \\
\hline Lungs & Pulmonary edema caused by phlegm dominance [35], Cold and wet dystemperament of lungs [46] \\
\hline Uterus & - \\
\hline Spleen & Cold dystemperament of spleen [22] \\
\hline Drugs and toxins & - \\
\hline Environmental factors & - \\
\hline Others & Fullness due to phlegm dominance [30] \\
\hline
\end{tabular}


has different characteristics [30, 33-35]. After interviewing 16 ITM specialists for 41 hours, the criteria for diagnosing thirst were reported: the amount of cousumed water and fluids, and change in tendency towards drinking water. Consumption of water based on habit and advice, suppression of thirst and drinking fluids to help relieve dry mouth were determined as the differential diagnosis of increased and decreased thirst. In the final analysis stage, a functional definition of thirst was obtained.

\section{Discussion}

In Iranian medical books, thirst is defined as the need for a cold and wet substance [21]; a strong desire for food of this quality is also considered as thirst [34]. In conventional medicine, thirst is a feeling that prioritizes receiving water $[36,37]$. The degree of thirst from the perspective of ITM depends on the individual's temperament, in addition to other individual and environmental factors [38]. Thirst and its decrease have been mentioned in most ITM books in the section related to diseases and symptoms of dystemperament, but in the section related to the symptoms of healthy temperament; sometimes the increase and decrease in thirst degree has been used to diagnose the dominant humor in a disease [30]. According to Hakim Jorjani, the causes of thirst are divided into two groups: bodily and non-bodily; bodily causes are divided into two groups: dystemperament and swelling/obstruction [39, 40].

Some scholars of ITM consider the "false thirst" as being thirsty due to the presence of a sticky sputum in the stomach, and the thirst due to the hot temperament of the stomach and liver and the thirst after eating the food is considered as "true thirst" [22]. Some Scholars belive that the thirst after eating, despite drinking enough water, is also a false thirst [28]. ITM specialists have also reported that the three factors recommended for the use of water by the Institute of Medicine (IOM) and the European Food Safety Authority (EFSA) are effective regardless of individual differences $[41,42]$, impaired thirst due to its suppression [43, 44] and dryness of the mouth [45] which must be distinguished from thirst and redcued thirst. The low number of studies on thirst and lack of access to some ITM specialists were some of the limitations of our study. It is recommended to investigate the prevalence of the symptom of increased and decreased thirst in patients and its relationship with the dominance of humors and dystemperament in future studies.

\section{Ethical Considerations}

Compliance with ethical guidelines

All ethical principles were considered in this article and this study has ethically approved (Code: IR.SBMU.RETECH.REC.1395.625). Participants were assured of the confidentiality of their information and were free to leave the study at any time.

\section{Funding}

The present paper was extracted from the $\mathrm{PhD}$ thesis of the first author, Department of Traditional Medicine, School of Traditional Medicine, Shahid Beheshti University of Medical Sciences.

Authors' contributions

Study Design, Conceptualization and Methodology: Morteza Mojahedi, Roshanak Mokaberinejad, Parisa Jafari ; Research, Original Writing: Parisa Jafari; Data Collection, Data Analysis and Interpretation: Parisa Jafari, Fatemeh Hakimi, Elham Parsa; Editing and Supervision: All authors.

Conflicts of interest

There is no conflict of interes in this sudy.

\section{Acknowledgements}

The authors would like to thank the Iranian Traditional Medicine specialists particpated in the study for their valuable cooperation. 
This Page Intentionally Left Blank 


\section{تحليل مفهوم عطش به عنوان يك علامت تشخيصى و علل آن از ديدَّاه طب ايرانى: يكى مطالعه بل روش هيبريد}

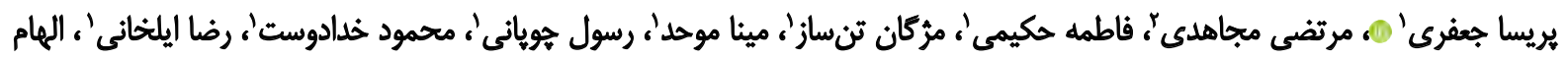

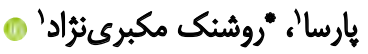

I. كروه طب سنتى، دانشكده طب سنتى، دانشكاه علوميرُشكى شهيد بهشتى تهران، ايران.

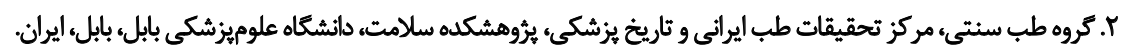

هدف تشنكى عاملى مؤثر در تنظيم مايعات بدن و علامت برخى ازئ بيمارىهاست. هدف از اين مطالعه إرائه تعريفى كاربردى از مفهوم

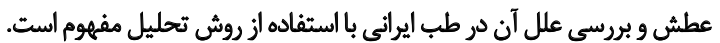

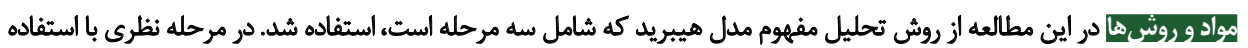

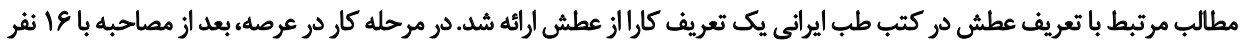

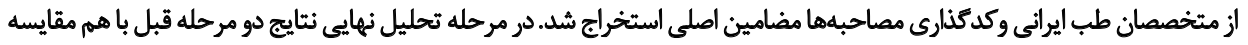

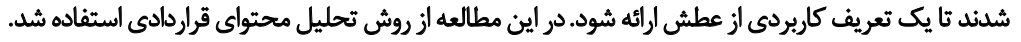

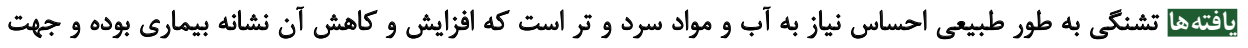

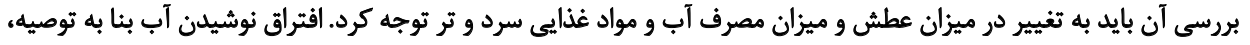

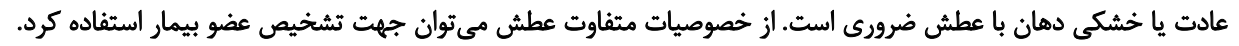

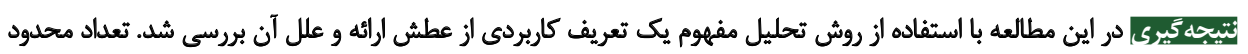

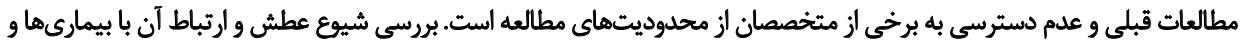

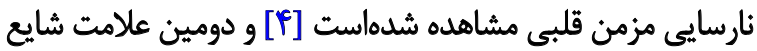

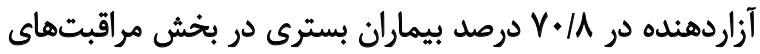

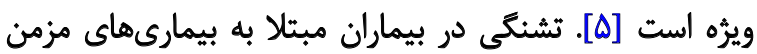

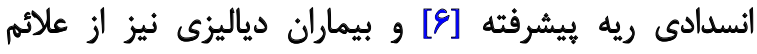

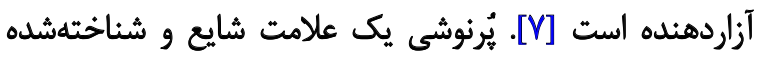

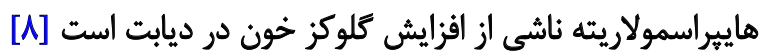

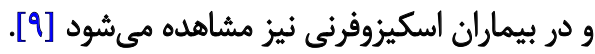

در كتب طب ايرانى نشانهها و علائم هر بيمارى بر اساس مشاهده

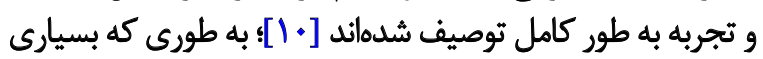

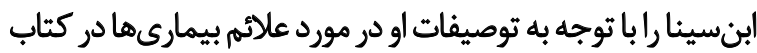

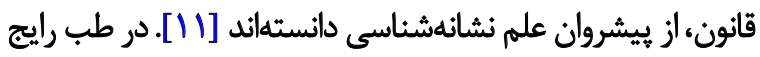

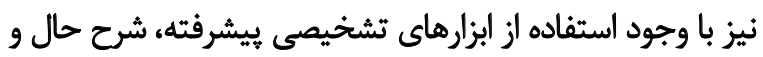

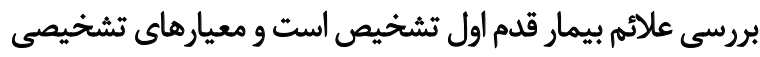

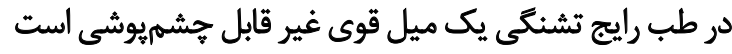

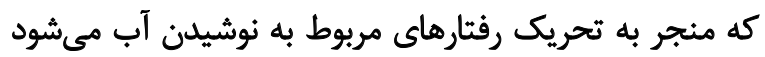

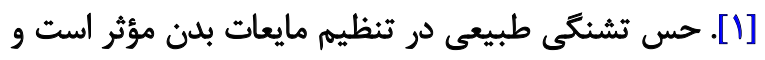

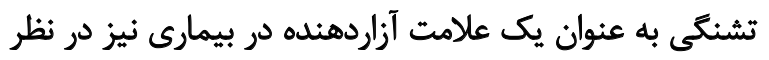

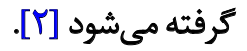

datos

جون تشنكى شاخصى از تغيير عملكرد طبيعى است كه خود

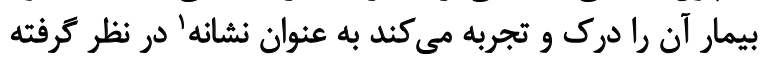

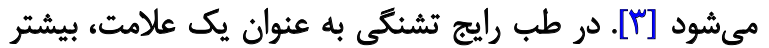

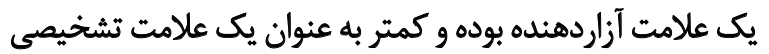

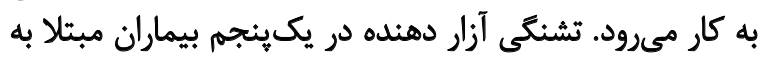

1. Symptom

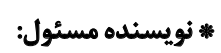

$$
\begin{aligned}
& \text { دكتر روشنك مكبرى نورئراد }
\end{aligned}
$$

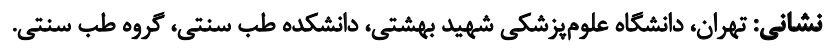

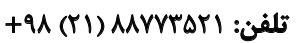

$$
\begin{aligned}
& \text { rmokaberi@gmail.com يست الكترونيكي: }
\end{aligned}
$$


است، اين علامت به عنوان مفهوم جهت مطالعه انتخاب شد.

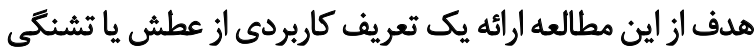

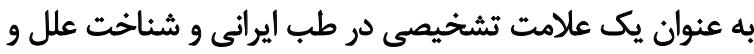

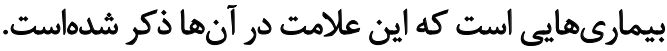

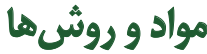

اين مطالعه يك مطالعه كيفى تحليل مفهوم به روش هيبريد،

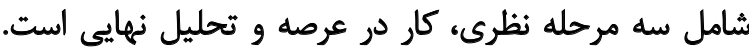

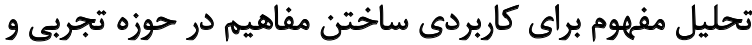

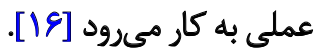
مرحله نظرى: هدف اين مرحله بررسى منابع موجود براى به دمست

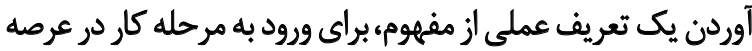

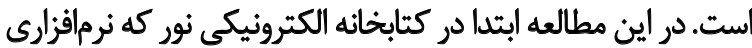

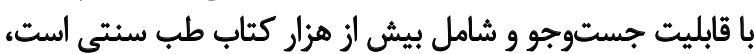

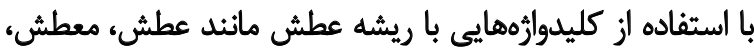

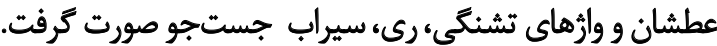
كتابهايى كه بيشتر به تعاريف و علل عطش برداخته بودند،

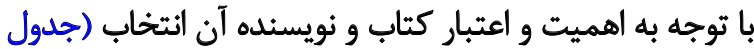

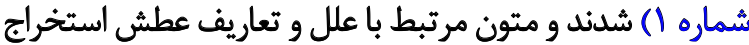

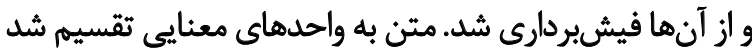

رمץ يكى از موارد موفق استفاده از شرح حال و علاتم ذكرشده

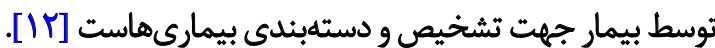
امروزه با توجه به شروع تحقيقات، تربيت دانشجو و تمايل به

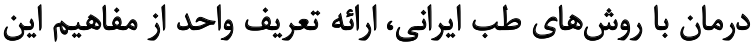

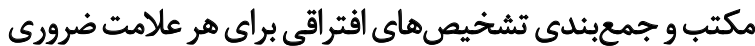

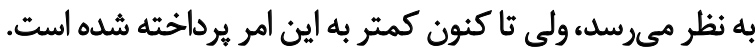
با توجه به توافق وايين متخصصان طب سنتى در تشخيص

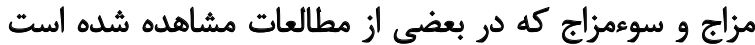

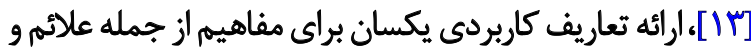

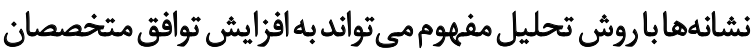
در تشخيص كمك كند. روش تحليل مفهوم يك تعرئ تعريف واضح و

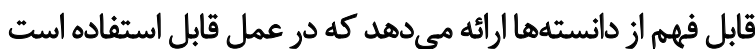

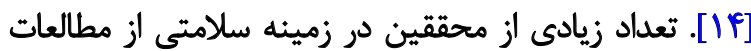

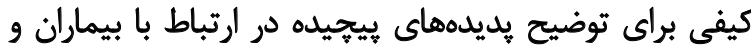

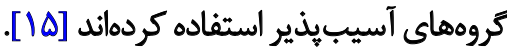
با توجه به اينكه در تقسيمبندى بيمارى ها در طب ايرانى بخش

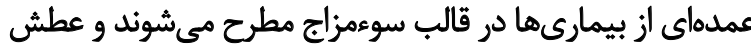

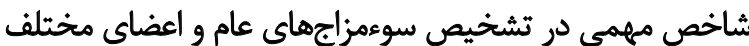

\section{Rome}

جدول ا. كتب موردبررسى در مرحله نظرى

\begin{tabular}{|c|c|c|c|c|}
\hline زبان & سال زندكى نويسنده (هجرى شمسى) & 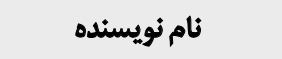 & ثام كتاب & شماره \\
\hline 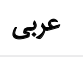 & ق ق قرن سوم & علىابنرين طبرى & فردوس الحكمه & 1 \\
\hline 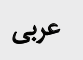 & قرن جهارم & خلف ابن عباس زهراوى & التصريف لمن عجز عن ثاليف & r \\
\hline عربى & قرن جهارم & مجوسى الهوازى & كامل الصناعه الطبيه & $r$ \\
\hline 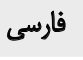 & قرن جهارم & اخوينى بخارى & هدايته المتعلمين & i \\
\hline 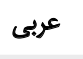 & قرن هنجم & ابن سيئا & القانون فى الطب & $\Delta$ \\
\hline عربى & قرن هفتم & ابن نفيس دمشقى & شح قائون اين سينا (شسح قرشى) & 8 \\
\hline عرئي & قرن هُتم & تفيس ابن عوض كرهاني & شرح الاسباب و العلامات & $\gamma$ \\
\hline 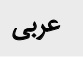 & قرن دهم & محمد ابن يوسف هروى & بحر الجواهر & $\wedge$ \\
\hline 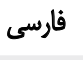 & قرن دوازدهم & عقيلى علوى شيرازى & خلاصه الحكمه & 9 \\
\hline 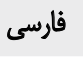 & قرن دوازدهم & عقيلى علوى شيرازى & ميخزن الادويه & 1. \\
\hline 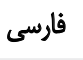 & قرن دوازدهم & محمد اكبر ارزانى (الكبر شاه) & ميزان الطب & 11 \\
\hline 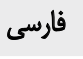 & قرن دوازدهم & محمد اكبر ارزانى (اكبر شاه) & مفرح القلوب & ir \\
\hline 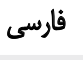 & قرن دوازدهم & محمد اكبر ارزانى (اكبر شاه) & طب اكيرى & ir \\
\hline 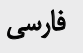 & قرن سيزدهم & اعظم خان جشتى & اكسير اعظم & If \\
\hline
\end{tabular}




\section{Ladil \\ يافتهنهاي نطرى}

تعريف عطش: از ديدكاه طب سنتى ايران كرسنكى نياز به

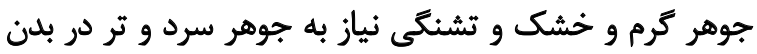

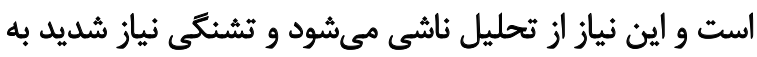

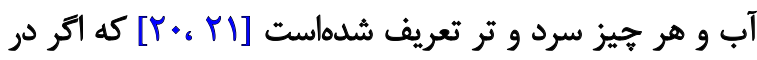

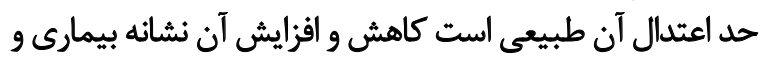

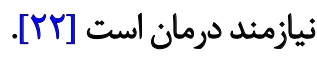

انواع عطشّ: در يك نوع تقسيم بندى عطش به دو نوع كاذب

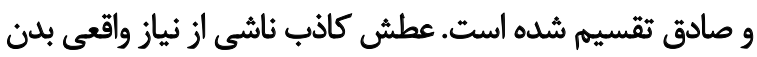

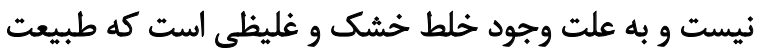

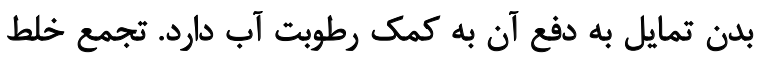

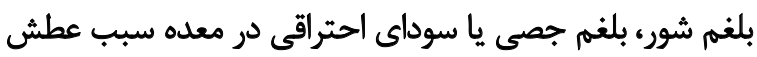

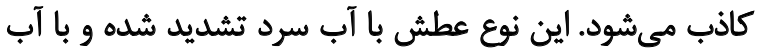

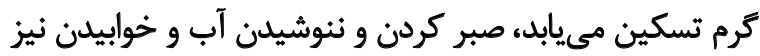

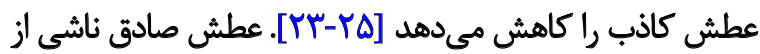

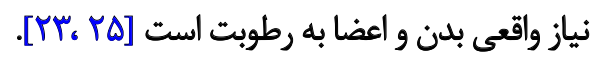

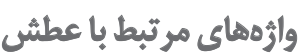

ا ـ معطش: يعنى عطش آورئده و داروييى است كه طبيعت بلن

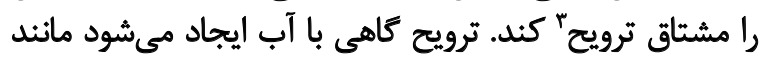

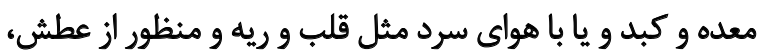

عطشصادق است نه كاذب [rع]

r. زئ مخالف عطش و به معنى سيراب شدن است [YV]. r. بيمارى عطش: جون عطش مفرط در اين بيمارى ديده

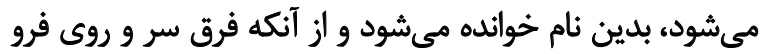

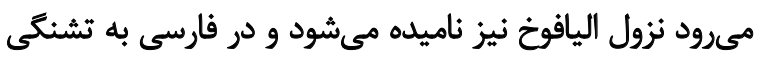

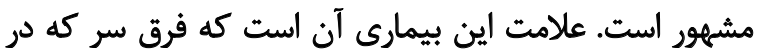

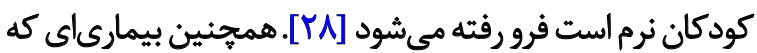

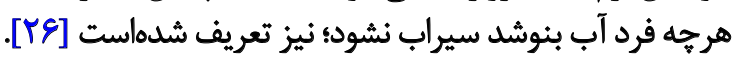

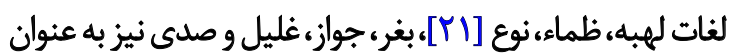

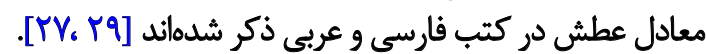
علل عطش: علل متعددى براي تشنكى در كتب طب ايراني

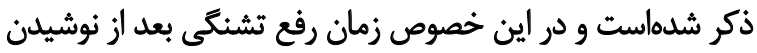

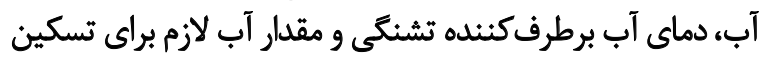

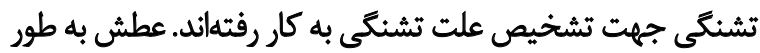

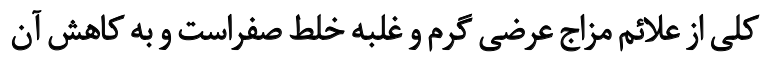

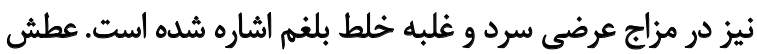
كاه علامت مرحله بحران يكى بيمارى است و كاه عرض برد به ساير ". ترويح به معنى آسوده كردن و آسايش دادن.
و مفاهيم آشكار و ينهان مشخص شد و مفاهيم بر اساس تعريف،

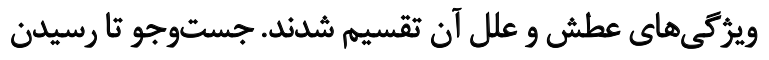

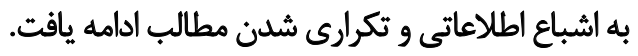

مرحله كار در عرصه: هدف از اين مرحله بررسي ويثركي ها و

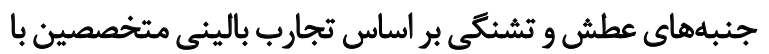
روش مصاحبه عميق بو ثاضمن واضحسازي مفاهيم و ويرّكي هاي تهني

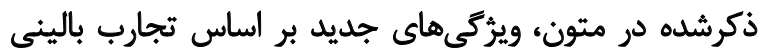

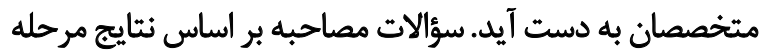

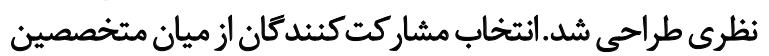

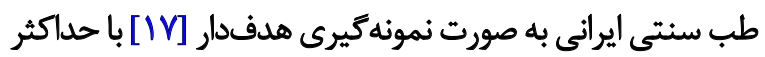
تنوع از نظر جنس، سن، سوابق و محيط كارى انجخام شد. مصاحبه

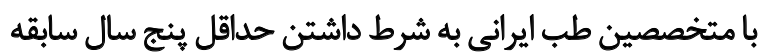

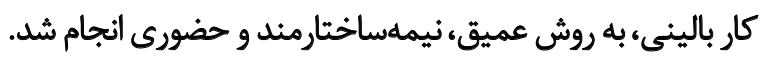

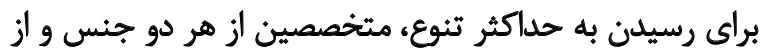

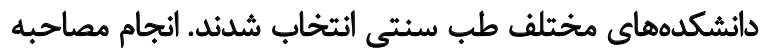

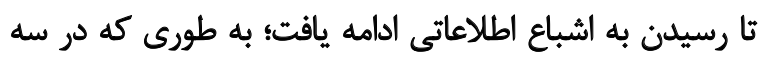

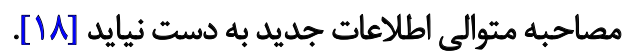

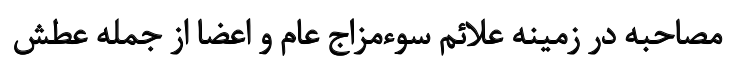

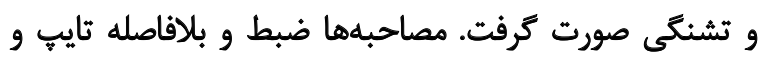

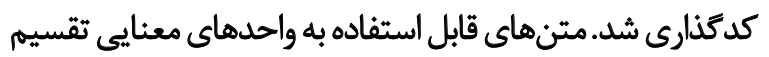
شدند و با استفاده از نرمافزار

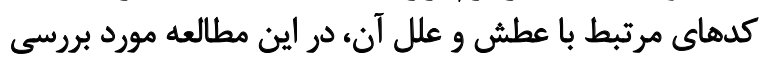

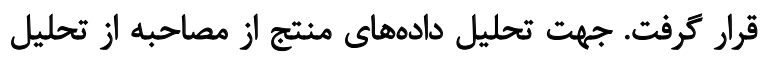

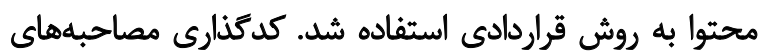

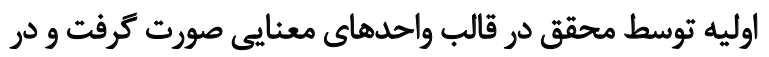
الدامه توسط تيم تحقيق كدهاى ابتدايى مورد بازنكرى قرار كرفت

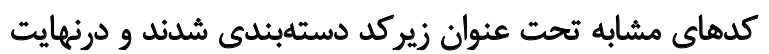

مضامين اصلى به دست آمد [19 مرحله تحليل نهايي:در مر حله تحليل نهايي،بامقايسه يافته هاي

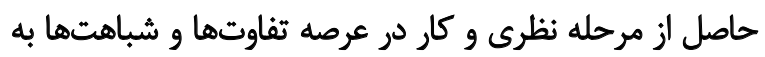

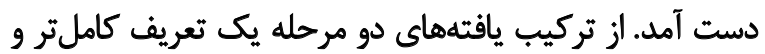
كاربردى از عطش و تشنكى طبق مبانى طب ايرانى ارائه شد.

جهت رعايت اعتمادسازى و تأييديذيرى، نتايج حاصل از

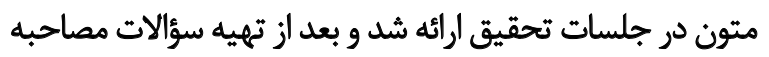

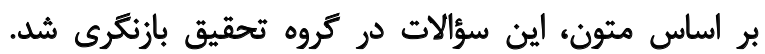

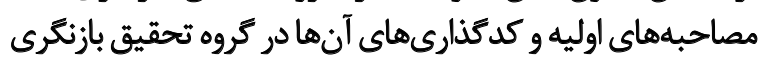

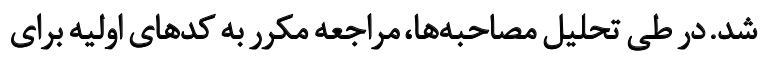

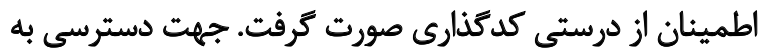

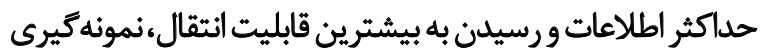
با حداكثر تنوع انجام شداث. 
جدول ب. بيمارىهايى كه افزايش عطش در آنها به عنوان علامت ذكر شده است

\begin{tabular}{|c|c|c|}
\hline 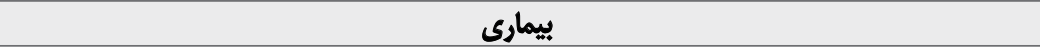 & عضو & شماره \\
\hline 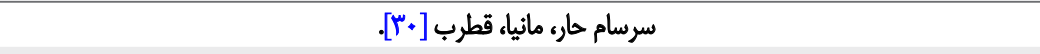 & (2) & 1 \\
\hline سوعمزاج كرم قلب [•"r] & قلب & $r$ \\
\hline 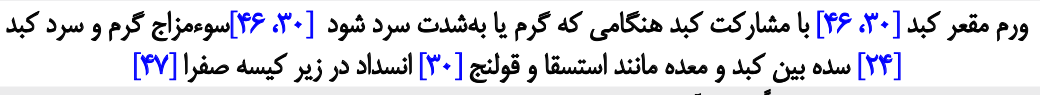 & كبد & $r$ \\
\hline 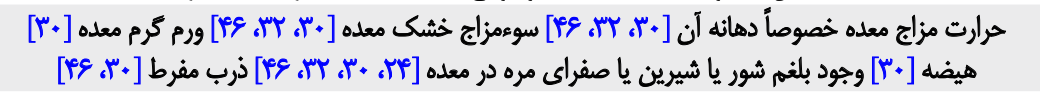 & ates & f \\
\hline 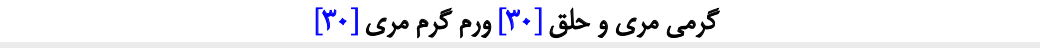 & مرى & $\Delta$ \\
\hline 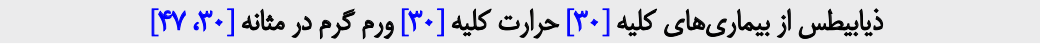 & كليه و مثائه & \& \\
\hline 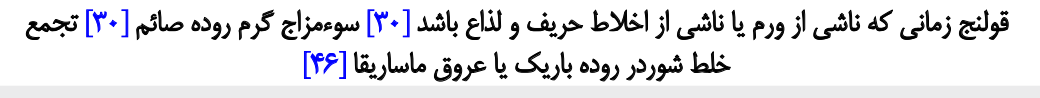 & امعاء & $\checkmark$ \\
\hline 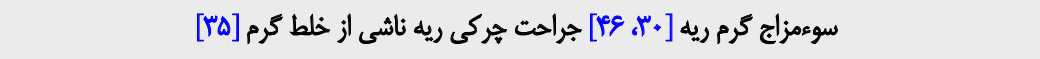 & ريه & $\wedge$ \\
\hline اختناق رحمه، قطع قاعدكى و اورام رحم [×"] & رحم & 9 \\
\hline 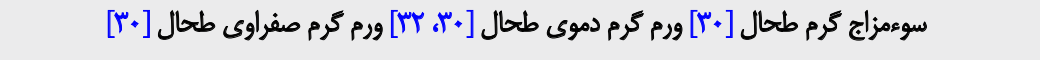 & طحال & 1. \\
\hline 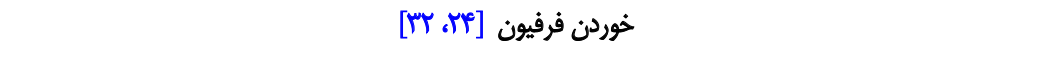 & داروها و سموم & 11 \\
\hline 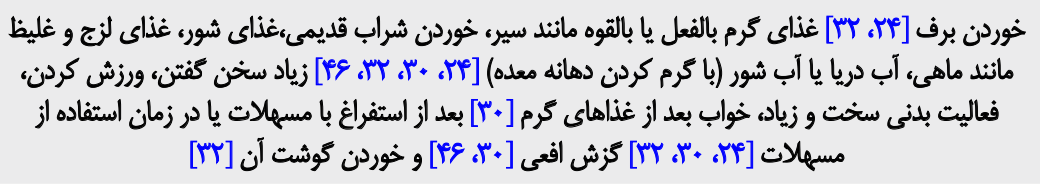 & 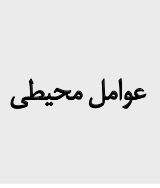 & ir \\
\hline 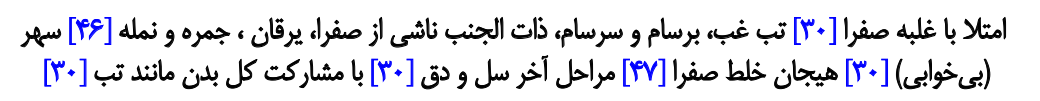 & ساير & ir \\
\hline
\end{tabular}

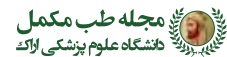

جدول ". بيمارىهايي كه كاهش عطش در آنها به عنوان علامت ذكر شده است.

\begin{tabular}{|c|c|c|}
\hline 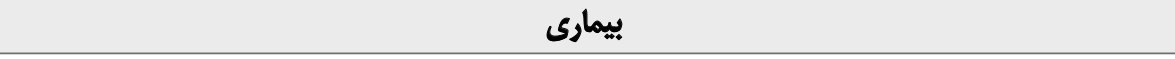 & 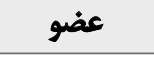 & شماره \\
\hline- & دماغ & \\
\hline- & 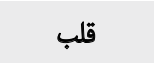 & \\
\hline 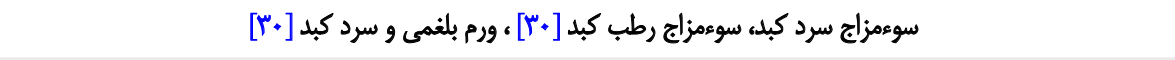 & 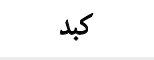 & \\
\hline 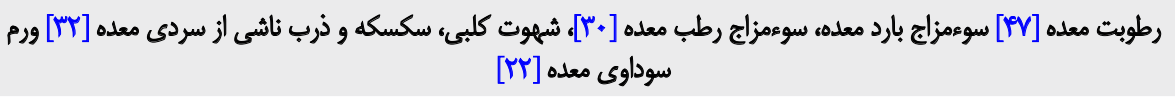 & معله & \\
\hline- & 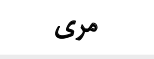 & \\
\hline سوءsزاج سرد كليه [ [TY] & كليه و مثانه & \\
\hline سردى امعاء [†] زحير بلغمى [Yr]] & امعاء & \\
\hline ورم ريه ناشى از خلط بلغم [هـ]، سوءمزاج سرد و رطب ريه [\&\&] & ري & \\
\hline- & 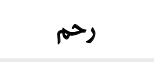 & \\
\hline 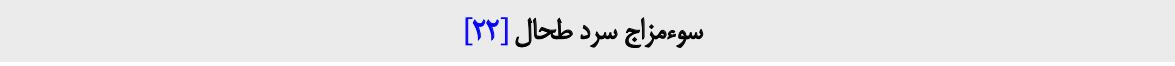 & 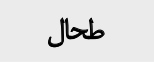 & \\
\hline- & 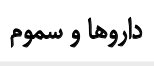 & \\
\hline- & عوامل محيطى - عل & \\
\hline امتلا با غلبه بلغم [ •"r] & ساير سير & \\
\hline
\end{tabular}

(2) 
كوارش ايجاد گردد بر عكس است و جه بسا استنشاق هواي سرد

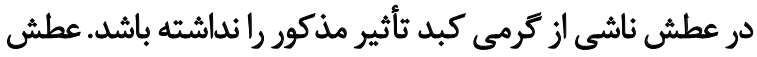

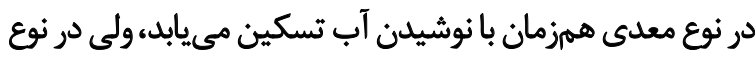

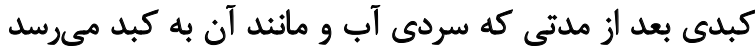

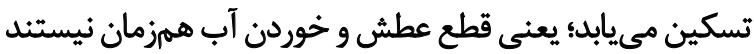

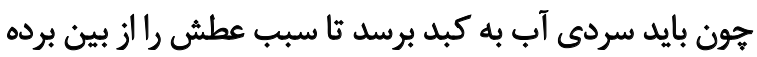

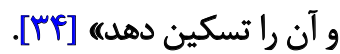

در عطش ناشى از سوءمزاج كرم كليه و بيمارى ذيابيطس

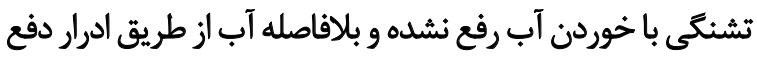

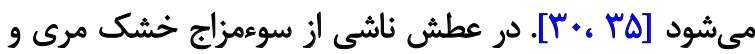

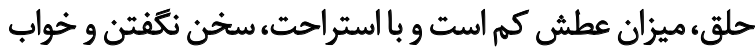

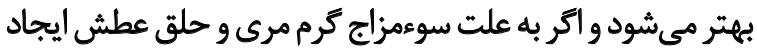

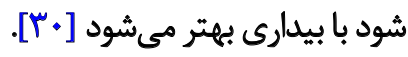

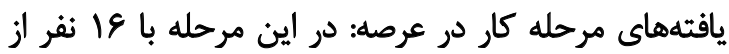

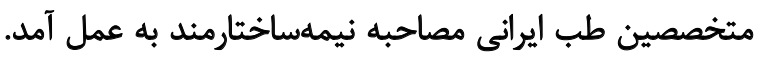

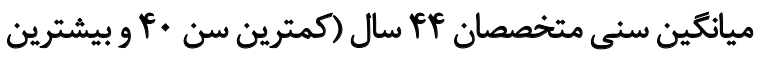

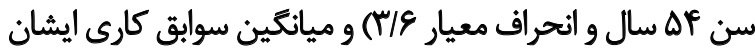

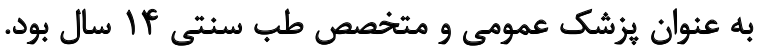

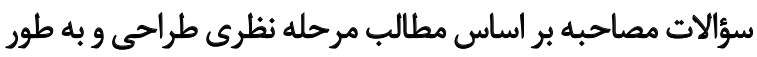

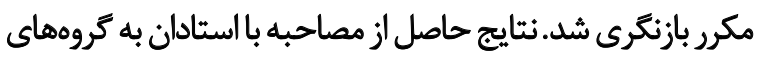

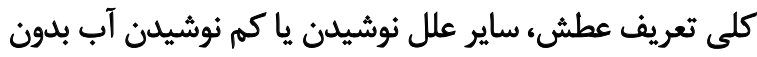

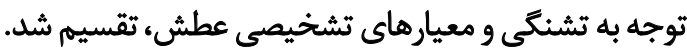

تعريف عطش: متخصصان طب سنتي ايرانى بيشتر عطش

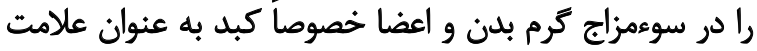

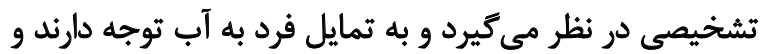

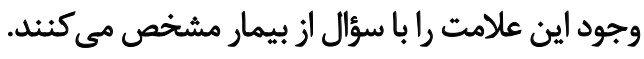

برخى از نظرات متخصصين شامل اين موارد است: عطش نوعى إنى

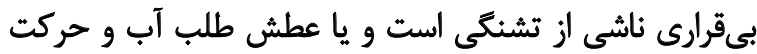

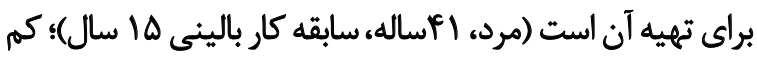

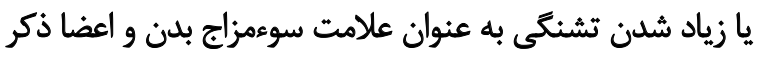

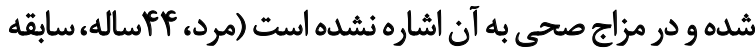

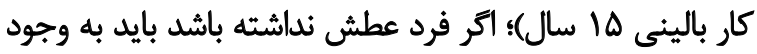

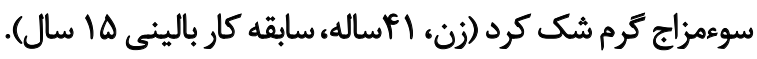

ساير علل نوشيدن يا كم نوشيدن آب بدون توجه به تشنكىى:

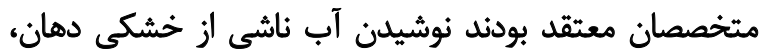

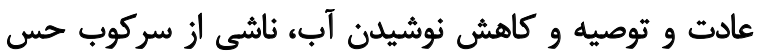

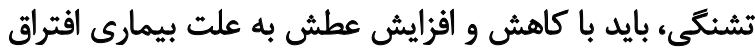

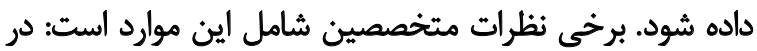

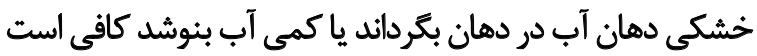

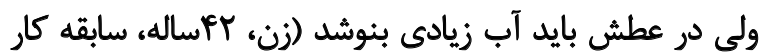
بالينى |r سال)؛

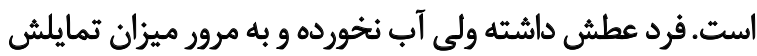

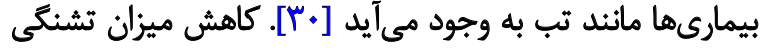

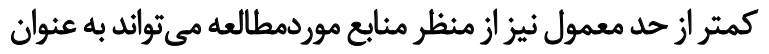

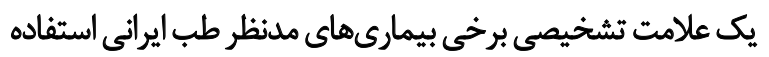

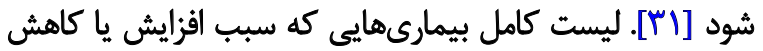
عطش مىشود در جدول هاى شماره ب و بار ارائه شده است است

$$
\text { مكائيسمهاى إيجاد عطش در طب ايرانى }
$$

مكانيسمهاي مختلفى براى به وجود آمدن عطش در كتب إنب

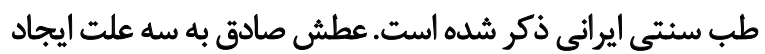

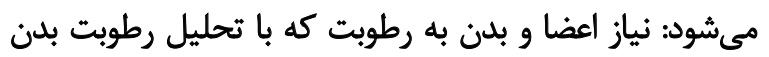

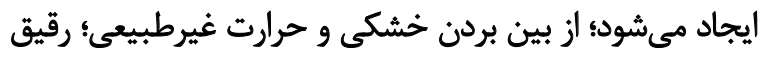

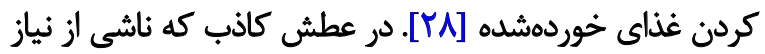

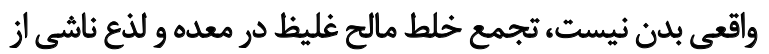

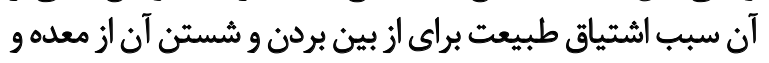

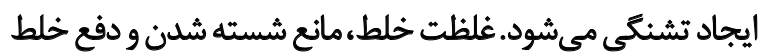

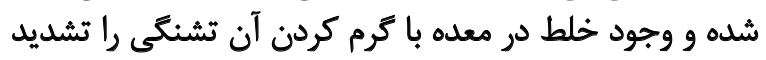

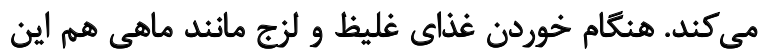

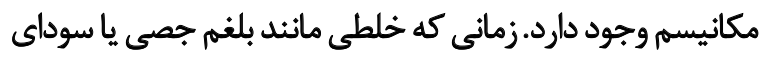

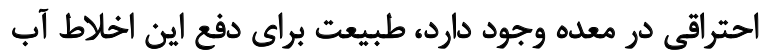

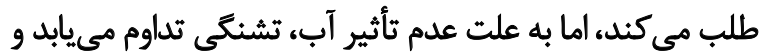

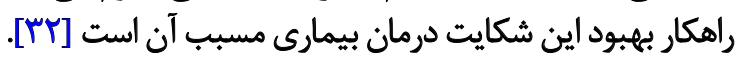

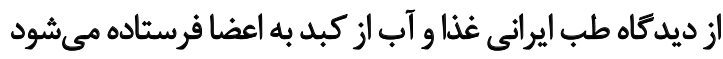

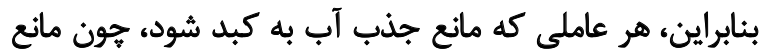

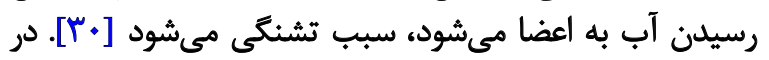

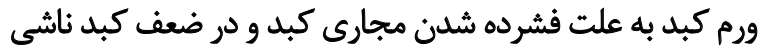

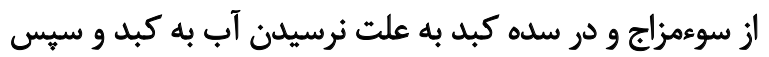
اعضا، عطش به وجود مى آيد كه با نوشيدن آب تسكيد آب به كبد ونميابد. تحليل رطوبات اصلى كه بعد از مصرف مسههلات و سموم ايجاد

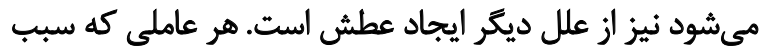

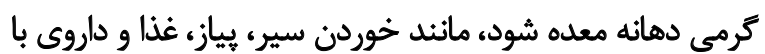

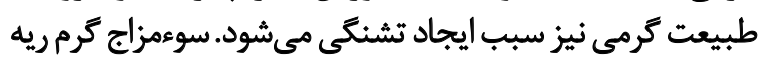

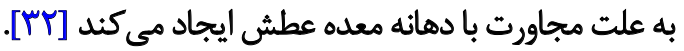

$$
\text { ويؤَى هاى عطش ناشى أز بيمارى هريك ازٔ اعضا }
$$

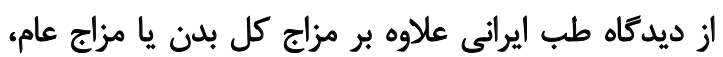

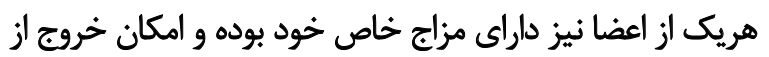

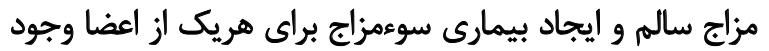

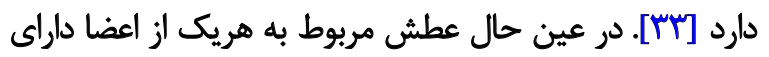

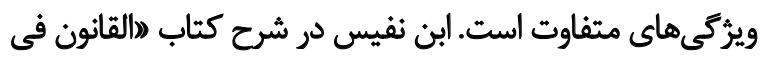

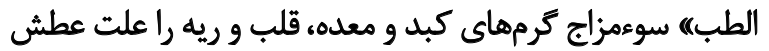

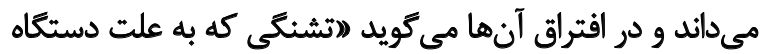

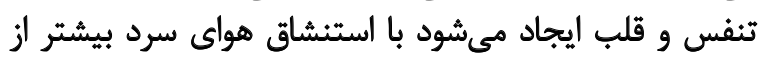

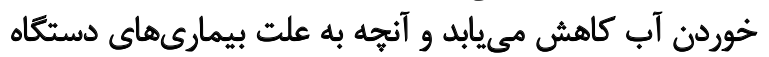




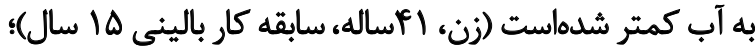

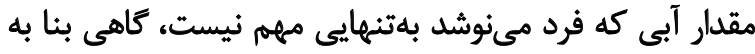
در كتب طب سنتى در تعريف تشنكي، اين مفهوم در رابطه با

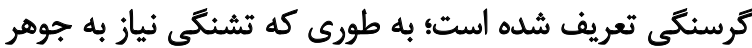

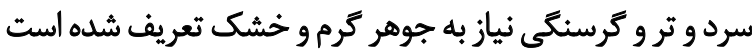

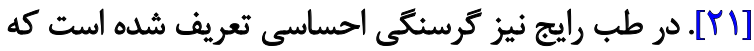

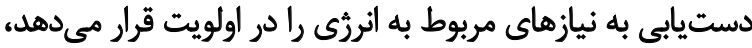

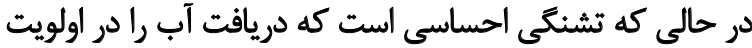

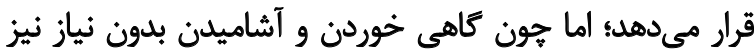

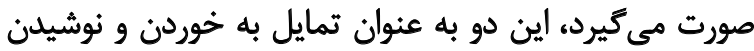

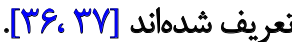

در طب ايرانى توجه به نياز به كيفيت تر و سرد در تعريف

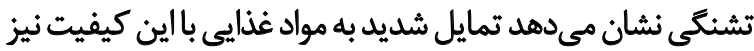

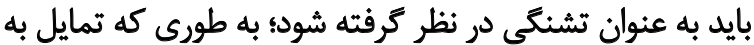

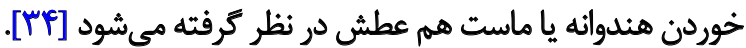
ميزان تشنكى و دريافت آب از ديدكاه دانشمندان طب ايراني

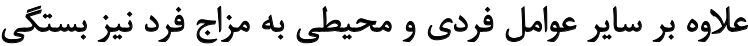

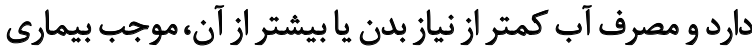

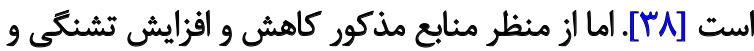

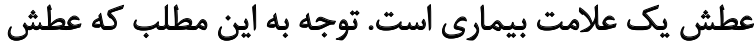

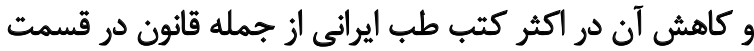

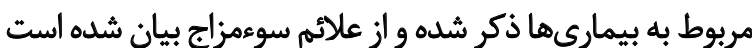

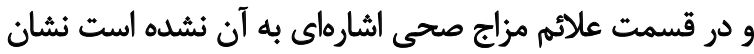

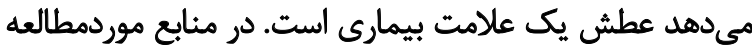

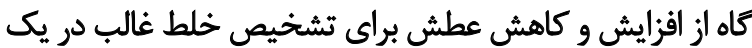

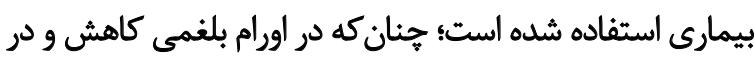
اورام صفراوى افزايش عطش بيان شدهاست [•rّ]. در يك مطالعه مرورى كه به مقايسه سبب تشنكى در طب بـ بري

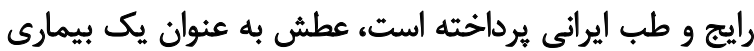

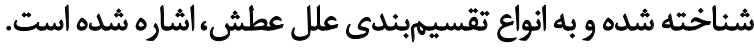

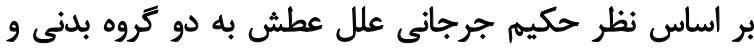

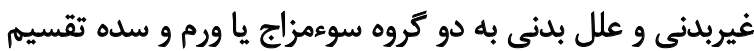

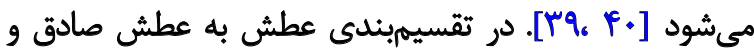

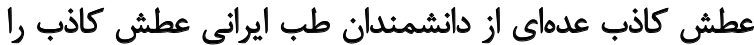

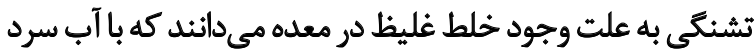

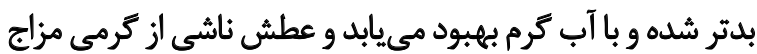

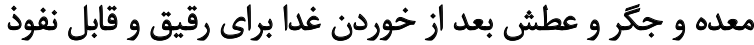

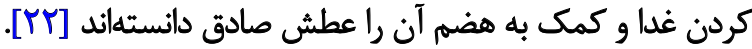
بعضى عطش بعد از خوردن غذا با وجود نوشيدن آن آب كان كافى راني رانيز

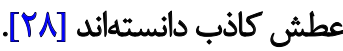

در بخش كار در عرصه اين مطالعه متخصصان طب ايرانى سه

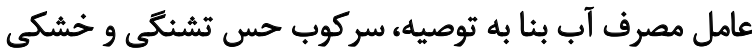

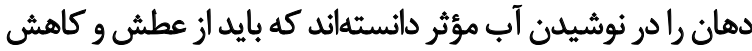

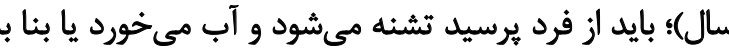

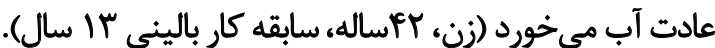

$$
\text { معيار تشخيصى عطش - مئ }
$$

براي تشخيص وجود عطش در بيمار، متخصصان از دو معيار

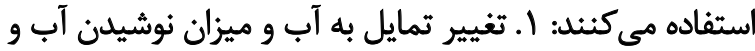

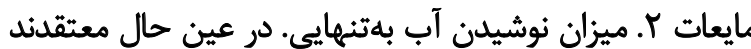
بايد به سن، شغل و شرايط محيطى فرد توجه شود.

بايد عطش بيمار نسبت به قبل سنجيده شود و اكر نسبت

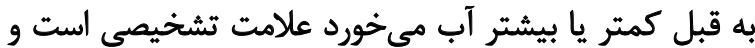

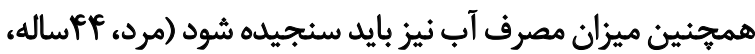

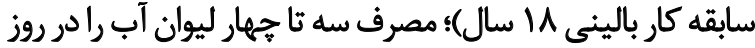

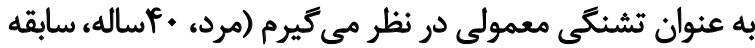

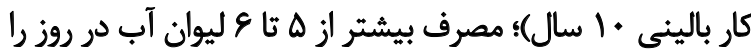

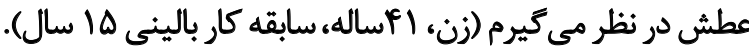
در يك جلسه گروهى با شركت بيش از • ل نفر از متخصصان

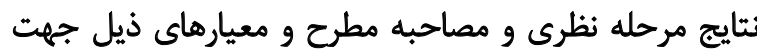

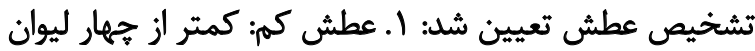

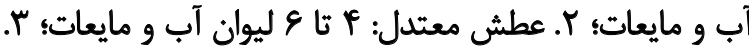

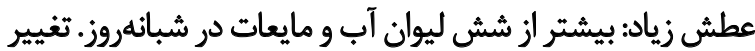

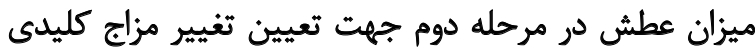

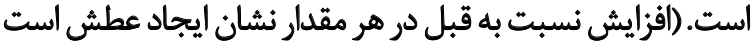

و كاهش نسبت به قبل در هر تعداد نشانه كمى عطش است است).

ياقتهها

از مقايسه دو مرحله نظرى و كار در عرصه يك تعريف عملكردى به شرح ذيل از عطش به دست آمد.

عطش احساس نياز به آب و مواد با كيفيت سرد و ثر است كه

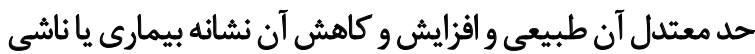

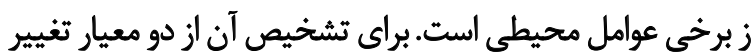

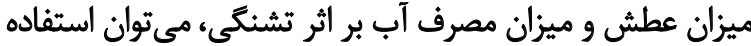

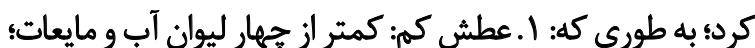

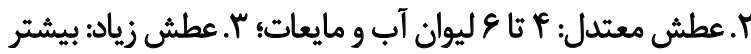

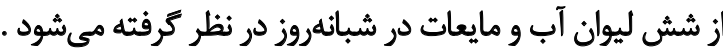

تغيير ميزان عطش در مرحله دوم جهت تعيين تغيير مزاج

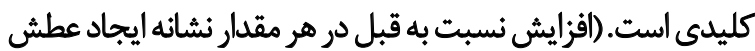

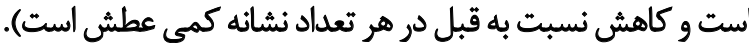

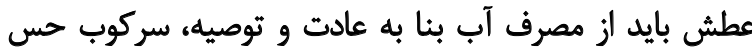
تشنكى و نوشيدن مايعات براي رفع خشكى دهان افت افتراق داده شود. 


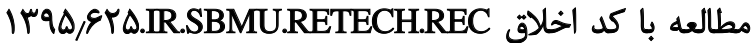

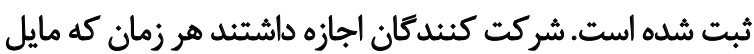

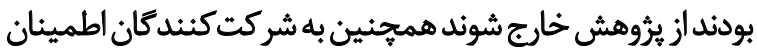
داده شده است كه اطلاعات آن ها محرمانه نثَه داشته مي شئشود.

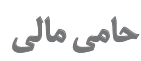

اين مطالعه بر كرفته از ياياننامه مقطع دكترى يريسا جعفرى

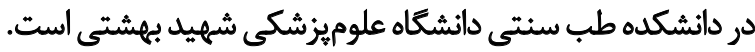

$$
\text { مشاركت ثويسند }
$$

مفهومسازى و روششناسى: يريسا جعفرى، مرتضى مجاهدى رونى

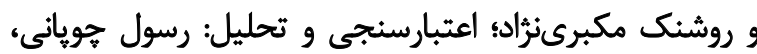

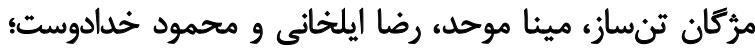

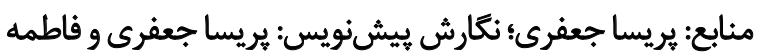

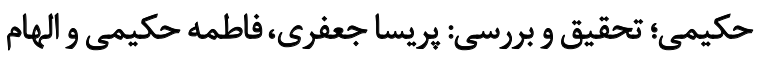
يارسا؛ ويراستارى و نهايى وسازى نوشته: همه نويسندكان.

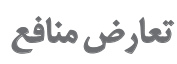

بنابر اظهار نويسندكان اين مقاله تعارض منافع ندارد.

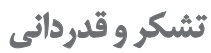

ضمن تشكر و قدردانى از دانشكده طب سنتى دانشكاه

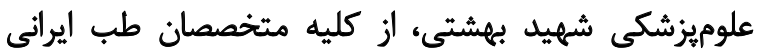

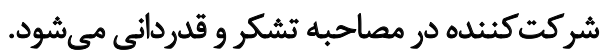

آن افتراق داده شود.

با بينكه وجود تأثير تفاوتهاي فردى مانند مشخصات فيزيكى،

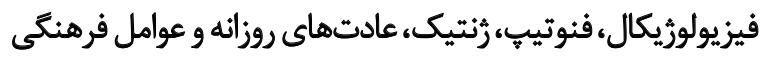

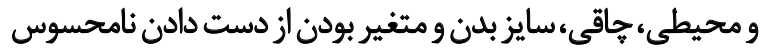

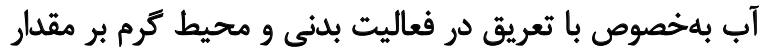

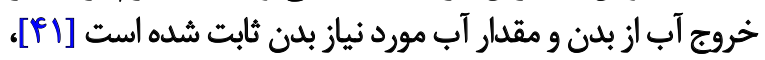

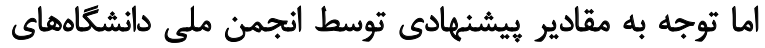

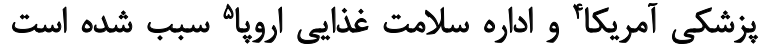

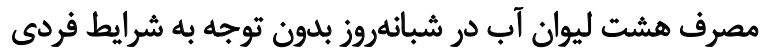

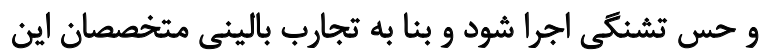
مورد بايد با عطش و تمايل واقعى به مصرف آب آب افتراق داده شود بندي

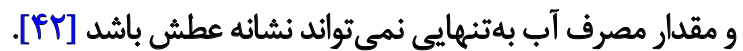
امكان ياسخ نامناسب و بهموقع به حس تشنكى در انسان و و

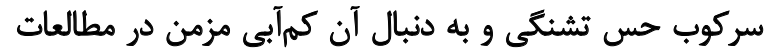

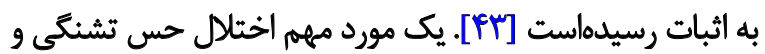

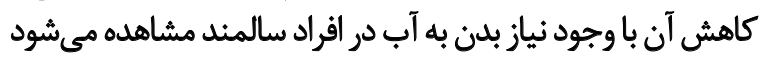

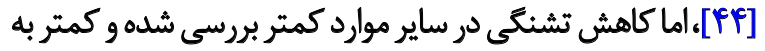

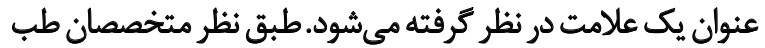

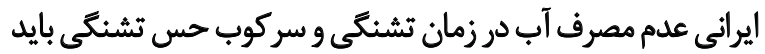

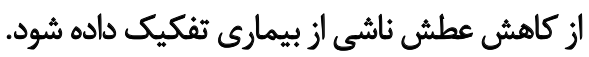
طبق نظر متخصصان، نوشيدن آب به علت خشكى دهان نيز

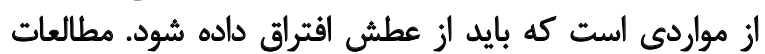

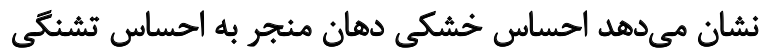

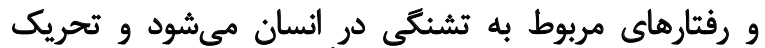

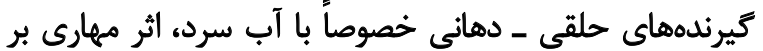

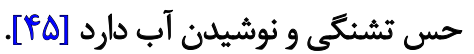
نتيجلمَيرى

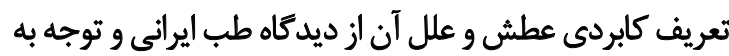

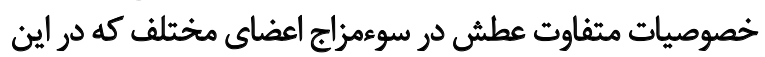

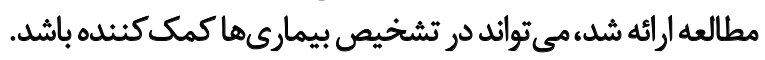

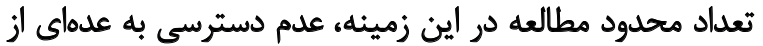

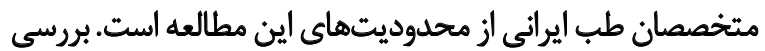

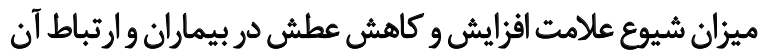

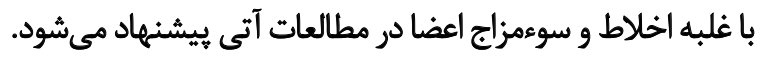

مالاحظات اخلاقي

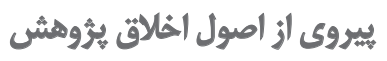

تمامى اصول اخلاقى در اين بروهش رعايت شده است و اين

5. The European Food Safety Authority (EFSA) 


\section{References}

[1] Allida SM, Hayward CS, Newton PJ. Thirst in heart failure: What do we know so far? Current Opinion in Supportive and Palliative Care. 2018; 12(1):4-9. [DOI:10.1097/SPC.0000000000000314] [PMID]

[2] Arai Sh, Stotts N, Puntillo K. Thirst in critically III patients: From physiology to sensation. American Journal of Critical Care. 2013; 22(4):328-35. [DOI:10.4037/ajcc2013533] [PMID] [PMCID]

[3] Lenz ER, Pugh LC, Milligan RA, Gift A, Suppe F. The middle-range theory of unpleasant symptoms: An update. Advances in Nursing Science. 1997; 19(3):14-27. [DOI:10.1097/00012272-199703000-00003] [PMID]

[4] Waldréus N, van der Wal MHL, Hahn RG, van Veldhuisen DJ, Jaarsma $\mathrm{T}$. Thirst trajectory and factors associated with persistent thirst in patients with heart failure. Journal of Cardiac Failure. 2014; 20(9):689-95. [DOI:10.1016/j.cardfail.2014.06.352]

[5] Puntillo K, Arai SR, Cooper BA, Stotts NA, Nelson JE. A randomized clinical trial of an intervention to relieve thirst and dry mouth in intensive care unit patients. Intensive Care Medicine. 2014; 40(9):1295-302. [DOI:10.1007/s00134-014-3339-z] [PMID] [PMCID]

[6] Gardiner C, Gott M, Payne Sh, Small N, Barnes S, Halpin D, et al. Exploring the care needs of patients with advanced COPD: An overview of the literature. Respiratory Medicine. 2010; 104(2):159-65. [DOI:10.1016/j. rmed.2009.09.007] [PMID]

[7] Fan WF, Zhang Q, Luo LH, Niu JY, Gu Y. Study on the clinical significance and related factors of thirst and xerostomia in maintenance hemodialysis patients. Kidney and Blood Pressure Research. 2013; 37(4-5):464-74. [DOI:10.1159/000355717] [PMID]

[8] Pendurthi AK, Karumanchi S. Clinical study of diabetic patients with special reference to their glycemic status. International Journal of Advances in Medicine. 2016; 3(2):166-70. [DOI:10.18203/2349-3933. ijam20160968]

[9] Perestrelo J, Teixeira B. Psychogenic polydipsia and hyponatremia - a side effect of psychosis: A review with a case report. Jornal Brasileiro de Psiquiatria. 2016; 65(3):300-3. [DOI:10.1590/0047-2085000000135]

[10] Farahi OR, Mozaffarpur SA, Saghebi R, Mojahedi M. The significance of tongue in traditional Persian medicine. Journal of Babol University of Medical Sciences. 2016; 18(8):73-9. [DOI:10.22088/jbums.18.8.73]

[11] Erolin C, Shoja MM, Loukas M, Shokouhi Gh, Rashidi MR, Khalili M, et al. What did Avicenna (Ibn Sina, 980-1037A.D.) look like? International Journal of Cardiology. 2013; 167(5):1660-3. [DOI:10.1016/j.ijcard.2012.09.178] [PMID]

[12] Schmulson MJ, Drossman DA. What is new in Rome IV. Journal of Neurogastroenterology and Motility. 2017; 23(2):151-63. [DOI:10.5056/ jnm16214] [PMID] [PMCID]

[13] Mojahedi M, Naseri M, Majdzadeh R, Keshavarz M, Ebadini M, Nazem $\mathrm{E}$, et al. Reliability and validity assessment of mizaj questionnaire: A novel self-report scale in Iranian traditional medicine. Iranian Red Crescent Medical Journal. 2014; 16(3):e15924. [DOI:10.5812/ircmj.15924] [PMID] [PMCID]

[14] Baldwin MA. Concept analysis as a method of inquiry. Nurse Researcher. 2008; 15(2):49-58. [DOI:10.7748/nr2008.01.15.2.49.c6329] [PMID]

[15] Erlingsson C, Brysiewicz P. A hands-on guide to doing content analysis. African Journal of Emergency Medicine. 2017; 7(3):93-9. [DOI:10.1016/j. afjem.2017.08.001] [PMID] [PMCID]

[16] Schwartz-Barcott D, Patterson BJ, Lusardi P, Farmer BC. From practice to theory: Tightening the link via three fieldwork strategies. Jour- nal of Advanced Nursing. 2002; 39(3):281-9. [DOI:10.1046/j.1365 2648.2000.02275.x] [PMID]

[17] Palinkas LA, Horwitz SM, Green CA, Wisdom JP, Duan N, Hoagwood K. Purposeful sampling for qualitative data collection and analysis in mixed method implementation research. Administration and Policy in Mental Health and Mental Health Services Research. 2015; 42(5):53344. [DOI:10.1007/s10488-013-0528-y] [PMID] [PMCID]

[18] Fusch PI, Ness LR. Are we there yet? Data saturation in qualitative research. The Qualitative Report. 2015; 20(9):1408-16. https://www. researchgate.net/publication/282955844

[19] Bengtsson M. How to plan and perform a qualitative study using content analysis. NursingPlus Open. 2016; 2:8-14. [DOI:10.1016/j. npls.2016.01.001]

[20] Ibn Sina HIA. [Al-Shifa (the book of healing) (Arabic)]. Zayed S, editor. Qom: Libraries of Mar'ashi-e-Najafi; 1984. http://lib.ahlolbait.ir/parvan/ resource/87366/

[21] Al-Harwi MIY. [Sea of jewels (Bahr al-Jawahir) (Persian-Arabic)] Qom: Jalal al-Ddin; 2008. http://opac.nlai.ir/opac-prod/bibliographic/2042899

[22] Nazem Jahan MA. [Exir-e azam (great elixir) (Persian)]. Tehran: Research Institute for Islamic \& Complementary Medicine; 2004. http:// opac.nlai.ir/opac-prod/bibliographic/2346832

[23] Al-Akhawyni Bokhari ABRIA. [Hidayat al-Muta`allemin fi al-Tibb (An educational guide for medical students) (Persian)]. Matini J, editor. Mashhad: Ferdowsi University of Mashhad; 1992. http://opac.nlai.ir/ opac-prod/bibliographic/530527

[24] Hakim Kermani NIA. Teb-e-Akbari (Akbari's medicine) [MA. Arzani, Persian trans]. Ehya-e Teb-e Tabiee, editor. Qom: Jalal al-Ddin; 2008. http://opac.nlai.ir/opac-prod/bibliographic/2042852

[25] Aghili Khorasani Shirazi MH. [Kholassat Al-Hekmah (the principal's of traditional Iranian medicine) (Persian)]. Nazem E, editor. Qom: Esmaeilian; 2006. http://opac.nlai.ir/opac-prod/bibliographic/1080323

[26] Aghili Khorasani Shirazi MH. [Makhzan al-advieh (the storehouse of medicaments) (Persian)]. Tehran: Tehran University of Medical Sciences; 2009. http://opac.nlai.ir/opac-prod/bibliographic/1794655

[27] Dehkhoda AA. [Dehkhoda dictionary (Persian)]. Tehran: University of Tehran Press; 2010. http://opac.nlai.ir/opac-prod/bibliographic/2398662

[28] Shah Arzani MAIM. [Mofarah al-Gholoob (fun of hearts) (Persian)]. Lahore: Eslamieh; 1915. http://opac.nlai.ir/opac-prod/ bibliographic/777841

[29] Arzani MA. [Mizan al-tib (a medical handbook) (Persian)]. Qom: Sama; 2001. http://opac.nlai.ir/opac-prod/bibliographic/1133121

[30] Ibn Sina. [Al-Qānūn fi al-Ṭibb (the canon of medicine) (Arabic)]. Beirut: Dar al-Ahya Al-Toras al-Arabi; 2005. http://lib.eshia.ir/73052/1/17

[31] Parsa E, Mojahedi M, Chaichi Raghimi M, Ilkhani R, Zareiyan A, Mokaberinejad $R$, et al. A review of the indices of mizaj-e-meda (temperament of stomach) identification in Persian medicine. Journal of Babo University of Medical Sciences. 2018; 20(7):63-70. http://jbums.org/ article-1-7489-en.html

[32] Kermani NIA. [Sharh al-Asbab va al-Alamat (explaining the causes and signs) (Arabic)]. Ehya-e Teb-e Tabiee, editor. Tehran: Jalal al-Ddin; 2008. http://opac.nlai.ir/opac-prod/bibliographic/2042845 
[33] Salmannegad H, Mojahedi M, Mozaffarpur SA, Saghebi R. The review of indices of mizaj-e-damagh (temperament of brain) identification in Persian medicine. Journal of Babol University of Medical Sciences. 2016; 18(11):71-9. http://jbums.org/article-1-6157-en.html

[34] Ibn al-Nafis. [Sharh Qanon-e Ibn Sina (a commentary on Avicenna's canon) (Arabic)]. Tehran: Research Institute for Islamic \& Complementary Medicine; 2011. http://centlib.iums.ac.ir:8800/site/catalogue/128932

[35] Al-Zahrawi A. [Al-Tasrif le Man Ajiza an al-Ta'lif (the Kitab al-Tasrif) (Arabic)]. Tehran: Iran University of Medical Sciences; 2009. http://opac.nlai. ir/opac-prod/bibliographic/2498430

[36] Mattes RD. Hunger and thirst: Issues in measurement and prediction of eating and drinking. Physiology \& Behavior. 2010; 100(1):22-32. [DOI:10.1016/j.physbeh.2009.12.026] [PMID] [PMCID]

[37] McKiernan F, Hollis JH, McCabe GP, Mattes RD. Thirst-drinking, hunger-eating; tight coupling? Journal of the American Dietetic Association. 2009; 109(3):486-90. [DOI:10.1016/j.jada.2008.11.027] [PMID] [PMCID]

[38] Jafari P, Mojahedi M, Zareiyan A, Mokaberinejad R, Chaichi Raghimi $M$, Hakimi F, et al. Water intake from the points of view of Rhazes and Avicenna. Complementary Medicine Research. 2019; 26(2):126-32. [DOI:10.1159/000491706] [PMID]

[39] Gorgani I. [Zakhireye Khwarazmshahi (treasure of Khwarazmshahi) (Persian)]. Tehran: Iranian Academy of Medical Sciences; 2001. http:// opac.nlai.ir/opac-prod/bibliographic/704603

[40] Amirian T, Maddahi SZ, Azadbakht M, Yousofpour M. [A comparative study on the views of Persian medicine and conventional medicine about thirst and its etiology (Persian)]. Journal of Mazandaran University of Medical Sciences. 2016; 26(139):246-57. http://jmums.mazums. ac.ir/article-1-8167-en.html

[41] Stanhewicz AE, Larry Kenney W. Determinants of water and sodium intake and output. Nutrition Reviews. 2015; 73(Suppl 2):73-82. [DOI:10.1093/nutrit/nuv033] [PMID]

[42] Gandy J. Water intake: Validity of population assessment and recommendations. European Journal of Nutrition. 2015; 54(Suppl. 2):11-6. [DOI:10.1007/s00394-015-0944-8] [PMID] [PMCID]

[43] Thornton SN. Thirst and hydration: Physiology and consequences of dysfunction. Physiology \& Behavior. 2010; 100(1):15-21. [DOI:10.1016/j. physbeh.2010.02.026] [PMID]

[44] Begg DP. Disturbances of thirst and fluid balance associated with aging. Physiology \& Behavior. 2017; 178:28-34. [DOI:10.1016/j.physbeh.2017.03.003] [PMID]

[45] Brunstrom JM. Effects of mouth dryness on drinking behavior and beverage acceptability. Physiology \& Behavior. 2002; 76(3):423-9. [DOI:10.1016/S0031-9384(02)00762-X]

[46] Al-Majusi AIA. [Kamel al-Sanaat al-Tibbiah (the perfect art of medicine) (Arabic)]. Ehya-e Teb-e Tabiee, editor. Tehran: Jalal al-Ddin; 2008. http://opac.nlai.ir/opac-prod/bibliographic/2042862

[47] Tabari AIS. [Firdous al-Hikmah (paradise of wisdom) (Arabic)]. Tehran: Research Institute for Islamic \& Complementary Medicine; 2008. 